\title{
A Discrete Geometric Approach to Solving 2-D Non-Linear Magnetostatic Problems
}

\author{
Paolo Bettini, Ruben Specogna, and Francesco Trevisan \\ Dipartimento di Ingegneria Elettrica, Gestionale e Meccanica (DIEGM), Università di Udine, Udine, Italy
}

\begin{abstract}
The aim of this paper is to introduce a Discrete Geometric Approach to solving 2-D non-linear magnetostatic problems. In particular, an efficient algorithm will be presented to solve magnetostatics in isotropic non-linear media by means of a Newton-Raphson scheme, in which the Jacobian is calculated analytically. Results on a reference configuration (TEAM Workshop Problem 25) are reported and discussed.
\end{abstract}

Index Terms-Cell method, discrete geometric approach, finite integration technique, non-linear magnetostatics, TEAM Workshop Problem 25.

\section{INTRODUCTION}

I N THIS PAPER, we focus on a Discrete Geometric Approach (DGA), based on the geometric structure behind Maxwell's equations [1]-[7], to solving non-linear magnetostatic problems in two-dimensional domains.

In particular, an efficient Newton-Raphson scheme will be presented in which the elements of the Jacobian matrix can be calculated analytically in the case of isotropic non-linear media, thanks to a proper choice of the magnetic constitutive matrix, which is constructed in a pure geometric way, by particularizing for triangles the ideas developed in [8]-[12] for tetrahedra.

As an application, a 2-D non-linear benchmark problem (TEAM Workshop Problem 25) has been considered, which consists in the numerical optimization of the shape of a die press, used for producing anisotropic permanent magnets, [13]. The optimal design is achieved by means of a search algorithm which combines a parallel genetic algorithm for global minimum search [14] with a deterministic algorithm for local refinement [15], coupled to the DGA algorithm which evaluates the objective function by solving the 2-D non-linear magnetostatic problem and return the relevant parameters to the search algorithm until a stop condition is reached.

\section{DisCRETE GEOMETRIC APPROACH}

In a domain of interest $D$, a pair of interlocked cell complexes $(\mathcal{K}, \tilde{\mathcal{K}})$ is introduced, [5], [16], where $\mathcal{K}$ is simplicial and $\tilde{\mathcal{K}}$ is obtained from it using the barycentric subdivision, see Fig. 1.

The interconnections between the pairs (edge $e$, node $n$ ), (face $f$, edge $e$ ) of $\mathcal{K}$ are described by the incidence matrices G, $\mathbf{C}$ respectively; we denote with $\tilde{\mathbf{C}}$ the incidence matrix between pairs (dual faces $\tilde{f}$, dual edges $\tilde{e}$ ) of $\tilde{\mathcal{K}}$ and $\tilde{\mathbf{C}}=\mathbf{C}^{T}$ holds. The edge and face vectors will be denoted in roman type $(\mathrm{e}, \mathrm{f})$.

Since TEAM Workshop Problem 25 is two dimensional, magnetic $\mathrm{H}$ and flux density $\mathrm{B}$ vector fields lying in a plane, we consider the projection on a plane of such a pair of interlocked grids, so the primal volumes coincide with the triangles of the mesh and the primal faces coincide with the edges of the mesh, see Fig. 2.

Manuscript received December 18, 2009; accepted February 21, 2010. Current version published July 21, 2010. Corresponding author: R. Specogna (e-mail: ruben.specogna@uniud.it).

Color versions of one or more of the figures in this paper are available online at http://ieeexplore.ieee.org.

Digital Object Identifier 10.1109/TMAG.2010.2044388

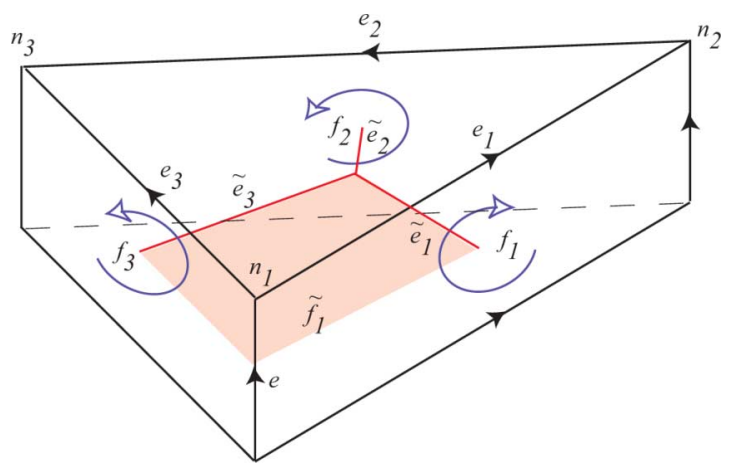

Fig. 1. Restriction of the primal and dual cell complexes is shown within a single volume $v$.

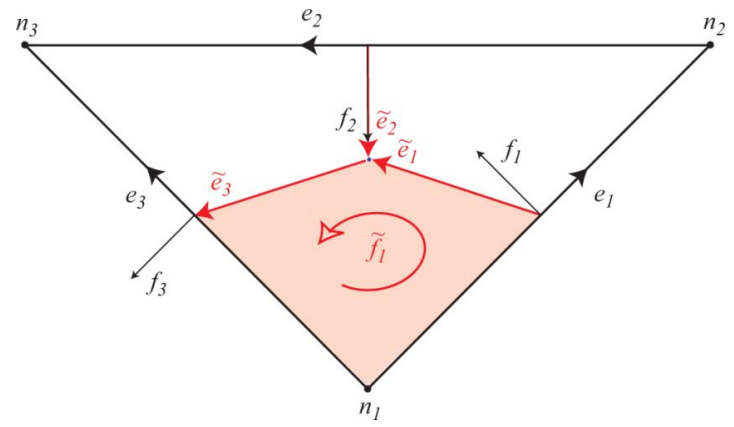

Fig. 2. In a 2-D domain, the primal volumes $v$ coincide with the triangles of the mesh and the primal faces $f_{i}$ coincide with the edges $e_{i}$ of the mesh.

Next, we consider the integrals of the field quantities involved in the magnetostatic problem with respect to the oriented geometric elements of $\mathcal{K}-\tilde{\mathcal{K}}$, yielding the Degrees of Freedom (DoF) arrays. There is a precise association between the DoFs and the geometric elements of $\mathcal{K}$ and $\tilde{\mathcal{K}}$ [17]. We denote by $\Phi$ the array of magnetic fluxes associated with primal faces $f$, by $\mathbf{F}$ the array of magneto-motive forces (m.m.f.s) associated with dual edges $\tilde{e}$, by $\mathbf{I}_{s}$ the arrays of electric source currents across dual faces $\tilde{f}$, and by $\mathbf{A}$ the array of circulations of the magnetic vector potential on primal edges $e$.

Maxwell's laws can be written exactly as topological balance equations between DoFs arrays, as

$$
\begin{aligned}
\left(\mathbf{C}^{T} \mathbf{F}\right)_{e} & =\left(\mathbf{I}_{s}\right)_{e}, \quad e \in D \\
(\mathbf{\Phi})_{f} & =(\mathbf{C A})_{f}, \quad f \in D
\end{aligned}
$$


where (1) is the Ampère's Law, (2) involves the array $\mathbf{A}$ in such a way that Gauss' Law $\mathbf{D} \boldsymbol{\Phi}=0$ is satisfied identically (since $\mathrm{DC}=0$ ). The discrete counterpart of the constitutive law $\mathrm{H}=$ $\nu \mathrm{B}$ can be written as

$$
\mathbf{F}=\boldsymbol{\Phi} \Phi
$$

where the matrix $\boldsymbol{\nu}$ is a square symmetric matrix which can be efficiently constructed in a pure geometric way, by particularizing for triangles the ideas developed in [7]-[11] for tetrahedra.

Considering the cell $v_{k}$ in Fig. $2, \mathrm{f}_{1}+\mathrm{f}_{2}-\mathrm{f}_{3}=0$ holds and the Gauss' law is always verified for an element-wise constant field B

$$
\Phi_{1}+\Phi_{2}-\Phi_{3}=\mathrm{B} \cdot \mathrm{f}_{1}+\mathrm{B} \cdot \mathrm{f}_{2}-\mathrm{B} \cdot \mathrm{f}_{3}=0 .
$$

Therefore the three fluxes are dependent and only two are used to produce the $\mathrm{B}$ field by

$$
\left[\begin{array}{ll}
\mathrm{f}_{1 x} & \mathrm{f}_{1 y} \\
\mathrm{f}_{2 x} & \mathrm{f}_{2 y}
\end{array}\right]\left[\begin{array}{l}
B_{x} \\
B_{y}
\end{array}\right]=\left[\begin{array}{l}
\Phi_{1} \\
\Phi_{2}
\end{array}\right]
$$

Inverting this linear system and using the fact that $\mathrm{f}_{i y}=\mathrm{e}_{i x}$ and $\mathrm{f}_{i x}=-\mathrm{e}_{i y}$ (see Fig. 2)

$$
\mathrm{B}=\frac{1}{2 S}\left[\begin{array}{ll}
\mathrm{e}_{2 x} & -\mathrm{e}_{1 x} \\
\mathrm{e}_{2 y} & -\mathrm{e}_{1 y}
\end{array}\right]\left[\begin{array}{l}
\Phi_{1} \\
\Phi_{2}
\end{array}\right]
$$

where $S$ is the area of $v_{k}$. The local constitutive matrix $\nu^{k}$, for an isotropic medium, can be obtained by

$$
\mathbf{F}^{k}=\boldsymbol{\nu}^{k} \boldsymbol{\Phi}^{k}=\left[\begin{array}{c}
\tilde{\mathrm{e}}_{1} \\
\tilde{\mathrm{e}}_{2} \\
\tilde{\mathrm{e}}_{3}
\end{array}\right] \nu \frac{1}{2 S}\left[\begin{array}{lll}
\mathrm{e}_{2 x} & -\mathrm{e}_{1 x} & 0 \\
\mathrm{e}_{2 y} & -\mathrm{e}_{1 y} & 0
\end{array}\right] \boldsymbol{\Phi}^{k} .
$$

Substituting (2) and (3) in (1), the set of algebraic equations governing the magnetostatic problem can be derived

$$
\mathbf{C}^{T} \boldsymbol{\nu} \mathbf{C A}=\mathbf{I}_{s} \text {. }
$$

\section{A. Non-Linear Case}

In the presence of non-linear (ferromagnetic) media, the following set of non-linear equations has to be solved:

$$
\mathbf{C}^{T} \boldsymbol{\nu}(\mathbf{A}) \mathbf{C A}=\mathbf{I}_{s} .
$$

An iterative scheme is needed to nullify the vector

$$
\mathbf{f}(\mathbf{A})=\mathbf{C}^{T} \boldsymbol{\nu}(\mathbf{A}) \mathbf{C A}-\mathbf{I}_{s} .
$$

At the $n$-th step of the iterative process we get

$$
\begin{aligned}
\mathbf{W}^{n} \mathbf{h}^{n} & =-\mathbf{f}\left(\mathbf{A}^{n}\right) \\
\mathbf{A}^{n+1} & =\mathbf{A}^{n}+\mathbf{h}^{n}
\end{aligned}
$$

where $\mathbf{h}^{n}$ is the correction vector used to determine the new estimate $\mathbf{A}^{n+1}$ and $\mathbf{W}^{n}$ is the Jacobian matrix or a non-singular matrix which approximates it, if a Newton-Rapshon or a Broyden method are adopted respectively. The advantage of the Broyden method is that the Jacobian matrix needs not to be recomputed at each iteration; on the other hand the order of convergence is lower (in between 1 and 2).

\section{B. Computation of the Jacobian}

The element $(i, j)$ in the Jacobian matrix can be written as

$$
\mathbf{W}_{i, j}=\frac{\partial \mathbf{f}_{i}}{\partial A_{j}}=\left[\mathbf{C}^{T} \boldsymbol{\nu}(\mathbf{A}) \mathbf{C}\right]_{i, j}+\left(\mathbf{C}^{T} \frac{\partial \boldsymbol{\nu}(\mathbf{A})}{\partial A_{j}} \mathbf{C A}\right)_{i}
$$

For the primal edges $e$ not belonging to the ferromagnetic region the second addendum of (12) vanishes while the first one is independent of $\mathbf{A}$. It is convenient to compute the contribution $\mathbf{W}^{k}$ to the Jacobian $\mathbf{W}$ due to the cell $v_{k}$. For isotropic non-linear media, the reluctivity matrix is scalar and the constitutive matrix $\nu^{k}$ can be rewritten as $\nu^{k}(\mathbf{A})=\mathbf{m}^{k} \nu^{k}\left(\mathbf{A}^{k}\right)$, where $\mathbf{m}^{k}$ is the matrix $\boldsymbol{\nu}^{k}$ calculated by swapping the reluctivity $\nu^{k}$ with the pure number 1 . The contribution of $v_{k}$ to the Jacobian becomes

$$
\mathbf{W}_{i, j}^{k}=\left[\mathbf{K}^{k}\right]_{i, j} \nu^{k}\left(\mathbf{A}^{k}\right)+\left(\mathbf{K}^{k} \mathbf{A}^{k}\right)_{i} \frac{\partial \nu^{k}\left(\mathbf{A}^{k}\right)}{\partial A_{j}^{k}}
$$

where $\mathbf{K}^{k}=\mathbf{C}^{k T} \mathbf{m}^{k} \mathbf{C}^{k}$ is a constant matrix. The derivative in (13) becomes

$$
\frac{\partial}{\partial A_{j}^{k}}\left(\frac{1}{\mu^{k}\left(\mathbf{A}^{k}\right)}\right)=-\frac{1}{\left(\mu^{k}\right)^{2}} \frac{\partial \mu^{k}}{\partial B^{k}} \frac{\partial B^{k}}{\partial A_{j}^{k}},
$$

where $B^{k}$ is the amplitude of the induction field in $v_{k}$, $\left(\partial \mu^{k}\right) /\left(\partial B^{k}\right)$ is the slope of the permeability curve.

By writing (2) for magnetic fluxes $\Phi_{1}$ and $\Phi_{2}$ associated with primal faces $f_{1}, f_{2}$ of the cell $v_{k}$ in Fig. 2 , and substituting it in (6), the components of B can be expressed in terms of $\mathbf{A}^{k}$ as

$$
\left[\begin{array}{l}
B_{x}^{k} \\
B_{y}^{k}
\end{array}\right]=\frac{1}{2 S}\left[\begin{array}{lll}
\mathrm{e}_{2 x} & -\mathrm{e}_{3 x} & \mathrm{e}_{1 x} \\
\mathrm{e}_{2 y} & -\mathrm{e}_{3 y} & \mathrm{e}_{1 y}
\end{array}\right] \mathbf{A}^{k} .
$$

By defining two vectors $\mathbf{k}_{x}$ and $\mathbf{k}_{y}$, such that

$$
\left[\begin{array}{l}
\mathbf{k}_{x} \\
\mathbf{k}_{y}
\end{array}\right]=\frac{1}{2 S}\left[\begin{array}{lll}
\mathrm{e}_{2 x} & -\mathrm{e}_{3 x} & \mathrm{e}_{1 x} \\
\mathrm{e}_{2 y} & -\mathrm{e}_{3 y} & \mathrm{e}_{1 y}
\end{array}\right]
$$

the amplitude of the flux density $B^{k}$ can be computed by

$$
B^{k}=\sqrt{\left(\mathbf{k}_{x} \cdot \mathbf{A}^{k}\right)^{2}+\left(\mathbf{k}_{y} \cdot \mathbf{A}^{k}\right)^{2}} .
$$

Using these equations, the $\left(\partial B^{k}\right) /\left(\partial A_{j}\right)$ term can be calculated analytically as

$$
\frac{\partial B^{k}}{\partial A_{j}^{k}}=\frac{\left(\mathbf{k}_{x} \cdot \mathbf{A}^{k}\right) k_{x j}+\left(\mathbf{k}_{y} \cdot \mathbf{A}^{k}\right) k_{y j}}{\sqrt{\left(\mathbf{k}_{x} \cdot \mathbf{A}^{k}\right)^{2}+\left(\mathbf{k}_{y} \cdot \mathbf{A}^{k}\right)^{2}}}
$$

where $k_{x j}$ and $k_{y j}$ represent the values in the $j$-th column of $\mathbf{k}_{x}$ and $\mathbf{k}_{y}$ respectively.

Finally (18) can be rewritten as

$$
\frac{\partial B^{k}}{\partial A_{j}^{k}}=\frac{B_{x}^{k} k_{x j}+B_{y}^{k} k_{y j}}{B^{k}}, j=1, \ldots, 3 .
$$

\section{NUMERICAL RESULTS}

The proposed approach has been applied to a magnetostatic non-linear benchmark problem (TEAM Workshop Problem 25), which consists in the numerical optimization of a die press, used for producing anisotropic permanent magnets, [13].

\section{A. Problem Description}

An overall view of the die press model is shown in Fig. 3. The die molds are set to form the radial flux distribution. The 


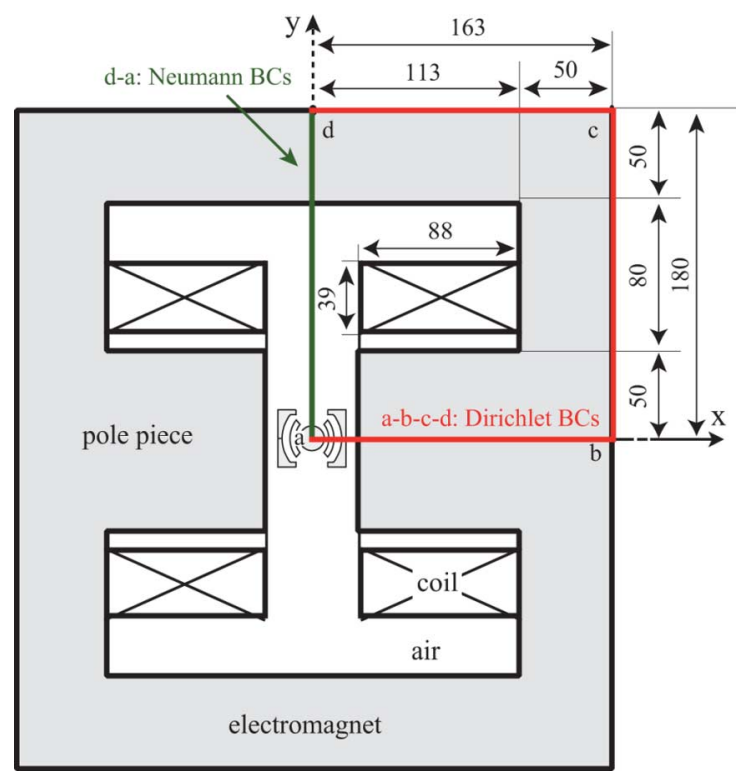

Fig. 3. TEAM Workshop problem 25: Sketch of the die press with the electromagnet; The draw is not to scale. Dirichlet BCs on a-b-c-d and Neumann BCs on d-a are imposed, respectively.

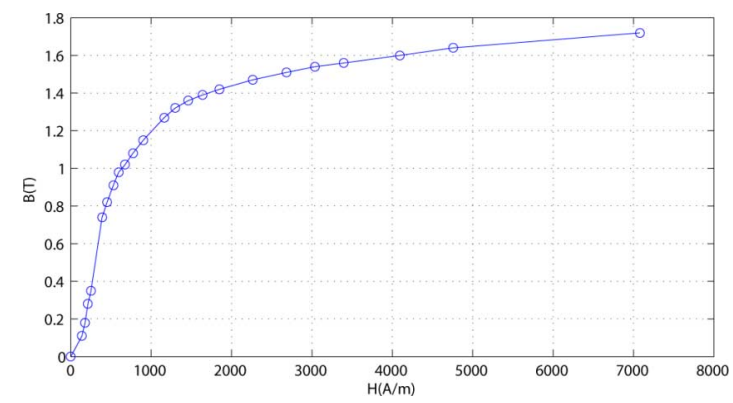

Fig. 4. TEAM Workshop problem 25: The B-H curve of steel used in the model for the die press and the electromagnet.

magnetic powder is inserted in the cavity. Both the die press and the electromagnet, for orientation of magnetic powder, are made of steel. The B-H curve used in the model is shown in Fig. 4; The B-H curve has been interpolated by using as interpolant a cubic spline. Dirichlet boundary conditions (BCs) are imposed on path a-b-c-d and Neumann BCs on path d-a, see Fig. 4.

The model can be assumed as two-dimensional and only one symmetric quarter of the structure is modeled. A detail of the benchmark geometry is shown in Fig. 5.

The shape of the inner die mold is assumed as a circle; The inside shape of the outer die mold is represented by the combination of an ellipse and a segment of a line parallel to the $x$-axis. Then, the radius $R_{1}$ of the inner die, the long and short axes $\left(L_{2}, L_{3}\right)$ of ellipse and the dimension $L_{4}$ are chosen as design variables, namely geometric constraints with specified upper and lower bounds.

In this paper we focus on the so-called Small Ampere-Turns reference case (4253 AT, dc current). The required induction field has radial direction, with $B_{x}$ and $B_{y}$ components along the line e-f in the cavity specified as follows

$$
\left\{\begin{array}{l}
B_{x}=0.35 \cos \theta \\
B_{y}=0.35 \sin \theta
\end{array}\right.
$$

where $\theta$ is the angle measured from the $x$-axis in Fig. 5 .

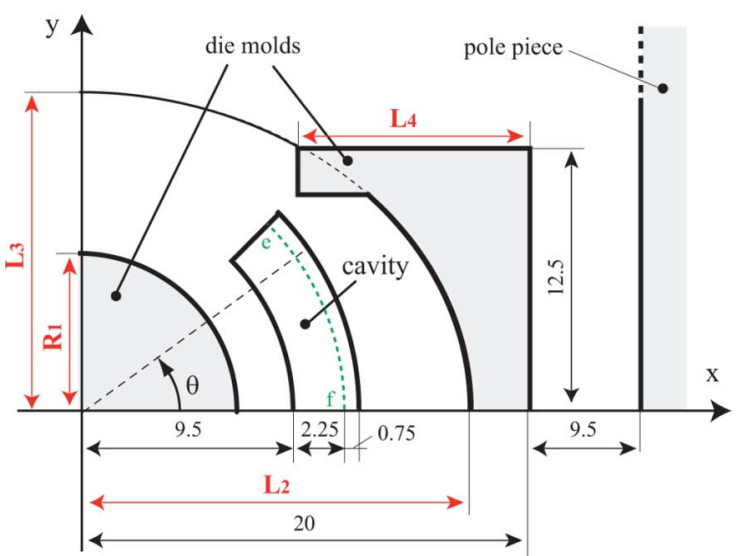

Fig. 5. TEAM Workshop problem 25: Enlarged view of a quarter of the structure. Numbers denote the given dimensions, whereas $R_{1}, L_{2}, L_{3}, L_{4}$ labels denote the design variables.

The objective function of the problem is given by

$$
W=\sum_{i=1}^{n}\left[\left(B_{x_{i}}^{c}-B_{x_{i}}^{o}\right)^{2}+\left(B_{y_{i}}^{c}-B_{y_{i}}^{o}\right)^{2}\right]
$$

where $n=10$ is the number of the control points $P_{i}$ selected along the path in the cavity; The superscripts " $\mathrm{c}$ " and "o" denote the calculated and reference values, respectively.

To asses the global quality of the final (optimal) induction field distribution, the following indexes (maximum error of the amplitude and of the angle $\varepsilon_{\theta}^{\max }$ of the flux density vector in $P_{i}$, $i=1, \ldots, n)$ are defined:

$$
\left\{\begin{array}{l}
\varepsilon_{B}^{\max }=\max \mid \frac{B_{i}^{c}-B_{i}^{o}}{B_{i}^{o}} \\
\varepsilon_{\theta}^{\max }=\max \left|\theta_{i}^{c}-\theta_{i}^{o}\right|
\end{array}\right.
$$

\section{B. Optimization Procedure}

The optimization procedure is based on a search algorithm, which combines a parallel genetic algorithm (GA), for global minimum search [14] and a deterministic algorithm (DA), for local refinement [15], coupled to the DGA algorithm, which evaluates the objective function (21) by solving a 2-D non-linear magnetostatic problem.

The final optimal design is achieved by means of an iterative scheme. Each point in the search space represents a different design, to be submitted from the search algorithm $(\mathrm{GA}+\mathrm{DA})$ to the DGA which returns the relevant parameters (fitness measure) to the search algorithm until a stop condition is reached. The GA is intrinsically parallel, and thus it has been efficiently compiled for execution on a parallel hardware using the OpenMP library.

The design variables are coded in strings using a Gray standard format and the operators, except minor details, are the classical ones proposed by Holland [18] and revised by Goldberg [14]. In particular, a roulette-wheel selection is adopted, based on string fitness values: the higher the fitness value of strings (individuals), the higher the probability of their copies in next generation; then, if $\left\{k_{1}\right\}$ are strings assigned at least one copy, $\left\{k_{2}\right\}$ are strings assigned at least two copies and so on, the new generation is created arranging first the $\left\{k_{N}\right\}$ strings, then $\left\{k_{N-1}\right\}$ strings and so on until the new population is completed. This arrangement is expected to favour the recombination between low performance elements by crossover and preserve strings with 


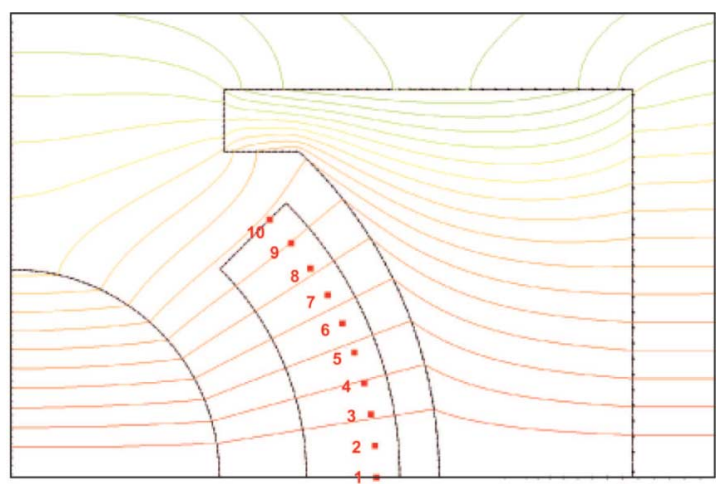

Fig. 6. TEAM Workshop problem 25: A detail of the die press model is shown. The distribution of the flux density, obtained with the final (optimal) shape of die molds, is shown.

best fitness values. As for mutation, a uniform probability distribution over each string is imposed.

Neither coupling restrictions nor "niche techniques" were applied, but an exponential fitness scaling mechanism is implemented. If $f(\mathbf{x})$ is the objective function, with $\mathbf{x}$ the design variable array, then the fitness function should be

$$
f_{f}(\mathbf{x})=e^{\alpha f(\mathbf{x})},
$$

being $\alpha$ a damping coefficient which can vary through generations, controlling the selective effect of the exponential function.

The final local minimum refinement is based on a well-established package for constrained optimization developed by Powell [15].

\section{Numerical Results}

The magnetic flux density distribution, evaluated for the final shape of the die molds obtained by means of the proposed optimization scheme, is shown in Fig. 6. In the cavity a radial distribution is clearly visible, as required.

A more quantitative assessment of the solution is provided by (22), which have been calculated on $n=10$ control points $P_{i}$ selected in the cavity. The maximum errors of the flux density in $P_{i}$ are $\varepsilon_{B}^{\max }=0.87 \%$ in terms of amplitude and $\varepsilon_{\theta}^{\max }=1.1$ deg in terms of direction.

The overall time of the optimization procedure relies mainly on the number of mesh elements, design variables, elements (individuals) of each population, and iterations. The number of DoFs for each problem, which coincides with the nodes of the considered mesh, is about 60000 , while the number of elements is about 130000 . The memory required to store this amount of unknowns was below $1 \mathrm{~Gb}$ for 4 threads simultaneously. The code, developed in Fortran 90, took approximately 10 seconds to perform a calculation of the 2-D non-linear magnetostatic solution on a double Dual-Core Intel ${ }^{\circledR}$ Xeon $^{\mathrm{TM}} 3.2 \mathrm{GHz}$ workstation with 8 GB RAM.

\section{CONCLUSION}

A Discrete Geometric Approach (DGA) to solving 2-D non-linear magnetostatic problems has been presented.
The main novelty relies in the development of an efficient Newton-Raphson scheme, in which the elements of the Jacobian matrix are calculated analytically for isotropic non-linear media, thanks to a proper choice of the magnetic constitutive matrix. As an application a 2-D non-linear benchmark problem (TEAM Workshop Problem 25) has been considered, which consists in the numerical optimization of the shape of a die press used for producing anisotropic permanent magnets [13].

\section{ACKNOWLEDGMENT}

The authors would like to thank F. Versolatto and M. Michelutti for the development of a preliminary version of the optimization algorithms.

\section{REFERENCES}

[1] Bossavit, "How weak is the weak solution in finite elements methods?," IEEE Trans. Magn., vol. 34, no. 5, pp. 2429-2432, Sep. 1998.

[2] T. Weiland, "Time domain electromagnetic field computation with finite difference methods," Int. J. Numer. Model., vol. 9, pp. 295-319, 1996.

[3] M. Clemens and T. Weiland, "Discrete electromagnetism with the finite technique," Progress In Electromagnetics Research (PIER) Monograph Series, vol. 32, pp. 65-87, 2001.

[4] E. Tonti, , A. Lichnerowicz and G. Ferrarese, Eds., "On the geometrical structure of elecromagnetism," in Gravitation, Electromagnetism and Geometrical Structures for the 80th Birthday. Bologna, Italy: Pitagora Editrice, 1995, pp. 281-308.

[5] E. Tonti, "Finite formulation of the electromagnetic field," IEEE Trans. Magn., vol. 38, no. 2, pp. 333-336, Feb. 2002.

[6] A. Bossavit and L. Kettunen, "Yee-like schemes on staggered cellular grids: A synthesis between FIT and FEM approaches," IEEE Trans. Magn., vol. 36, no. 4, pp. 861-867, Jul. 2000.

[7] A. Bossavit, Computational Electromagnetism. Brighton, MA: Academic, 1998.

[8] F. Trevisan and L. Kettunen, "Geometric interpretation of finite dimensional eddy current formulations," Int. J. Numer. Methods Eng., vol. 67, no. 13, pp. 1888-1908, 2006.

[9] R. Specogna and F. Trevisan, "Discrete constitutive equations in $A-\chi$ geometric eddy-currents formulation," IEEE Trans. Magn., vol. 41, no. 4, pp. 1259-1263, Apr. 2005.

[10] L. Codecasa and F. Trevisan, "Piecewise uniform bases and energetic approach for discrete constitutive matrices in electromagnetic problems," Int. J. Numer. Methods Eng., vol. 65, no. 4, pp. 548-565, 2006.

[11] L. Codecasa, R. Specogna, and F. Trevisan, "Symmetric positive-definite constitutive matrices for discrete eddy-current problems," IEEE Trans. Magn., vol. 43, no. 4, pp. 510-515, Apr. 2007.

[12] L. Codecasa, R. Specogna, and F. Trevisan, "Base functions and discrete constitutive relations for staggered polyhedral grids," Comput. Methods Appl. Mech. Engrg., vol. 198, pp. 1117-1123, 2009.

[13] N. Takahashi, K. Ebihara, K. Yoshida, T. Nakata, K. Ohashi, and K. Miyata, "Investigation of simulated annealing method and its application to optimal design of die mold for orientation of magnetic powder," IEEE Trans. Magn., vol. 32, no. 3, pp. 1210-1213, May 1996.

[14] D. E. Goldberg, Genetic Algorithm in Search, Optimization and Machine Learning. Reading, MA: Addison-Wesley, 1989.

[15] M. J. D. Powell, "Direct search algorithms for optimization calculations," Acta Numerica, vol. 7, pp. 287-336, 1998.

[16] E. Tonti, "Algebraic topology and computational electromagnetism," in Proc. 4th Int. Workshop Electr. Magn. Fields, Marseille, France, May 12-15, 1988, pp. 284-294.

[17] E. Tonti, "On the formal structure of physical theories," Quaderni dei Gruppi di Ricerca Matematica del CNR 1975.

[18] J. H. Holland, Adpatation in Natural and Artificial Systems: An Introductory Analysis With Application to Biology, Control, and Artificial Intelligence. Cambridge: MIT Press, 1992. 